Pure and Applied Mathematics Quarterly

Volume 9, Number 3

$487-492,2013$

\title{
Exemples de Groupes Semi-Simples Simplement Connexes Anisotropes Contenant Un Sous-Groupe Unipotent
}

\author{
Philippe Gille et Anne Quéguiner-Mathieu*
}

\begin{abstract}
We show that there are anisotropic groups of type $G_{2}$ (resp. $F_{4}$, $\left.E_{8}\right)$ containing a non-trivial smooth unipotent subgroup over a suitable field of characteristic 2 (resp. 3, 5).
\end{abstract}

Keywords : Galois cohomology, linear algebraic groups.

\section{Introduction}

Soit $k$ un corps d'exposant caractéristique $p$, et $G / k$ un groupe semi-simple. Dans cette note, on s'intéresse à la question naturelle suivante : à quelle condition le groupe $G$ contient-il un sous-groupe unipotent lisse et connexe non trivial?

Si le groupe $G$ est isotrope, l'existence d'un tel sous-groupe, que l'on peut en outre supposer déployé, est claire. Si de plus le corps de base $k$ est parfait, alors la réciproque est également vraie. En effet, un $k$-sous-groupe unipotent lisse non Received September 12, 2012.

MSC : 11E72, 20G05.

* Ce travail a bénéficié d'une aide de l'Agence Nationale de la Recherche portant la référence ANR-12-BL01-0005. 
trivial $U$ de $G$ donne lieu par [BT, 2.4,2.5] à un $k$-sous-groupe parabolique propre de $G$, ce qui impose que $G$ est isotrope. Ainsi, sur un corps parfait, l'isotropie est une condition nécessaire et suffisante à l'existence d'un tel sous-groupe.

Le cas intéressant est donc le cas des groupes anisotropes sur un corps non parfait. Dans ce contexte, l'équivalence précédente n'est plus vraie en général. Pour le voir, il suffit de considérer le groupe adjoint $G=\operatorname{PGL}_{1}(A)$, où $A$ est une algèbre simple centrale contenant un corps commutatif $K / k$. Si l'algèbre $A$ est à division, le groupe $G$ est anisotrope. De plus, il contient le $k$-sous-groupe $R_{K / k}\left(\mathbb{G}_{m}\right) / \mathbb{G}_{m}$, qui est unipotent et lisse dès que $K / k$ est purement inséparable. Un exemple explicite est donné par l'algèbre de présentation $X^{p}-X=x, Y^{p}=y$ et $X Y=Y(X+1)$ sur $\mathbb{F}_{p}(x, y)$, ou bien $\mathbb{F}_{p}((x))((y))$. En effet, cette algèbre est une algèbre cyclique de degré $p$ [GiS, $\S 2.5$, p. 36], à division par loc. cit. cor. 4.7.5, et elle contient une extension purement inséparable du centre, engendrée par $Y$.

Gopal Prasad a demandé s'il existait des groupes semi-simples simplement connexes ayant la même propriété. Dans cette note, nous répondons positivement à cette question en donnant des exemples de type $G_{2}, F_{4}$ et $E_{8}$ respectivement en caractéristique 2, 3 et 5, voir la Proposition 3.1. La contruction repose sur l'étude de l'invariant de Rost en caractéristique positive [GQM] que nous rappelons ici.

\section{L'invariant de Rost}

Si $G / k$ est un groupe semi-simple simplement connexe absolument presque $k$-simple, on dispose de l'invariant de Rost [EKLV] [GMS]

$$
r_{G}: H^{1}(k, G) \rightarrow H^{3}(k):=H^{3}(k,(\mathbb{Q} / \mathbb{Z})(2))
$$

qui associe à la classe d'un $G$-torseur une classe de cohomologie galoisienne de degré 3 , où pour $d \geq 0, H^{d+1}(k,(\mathbb{Q} / \mathbb{Z})(d))$ désigne le groupe de cohomologie galoisienne modifié à la Kato $[\mathrm{K}]$ sur la composante $l$-primaire si $k$ est de caractéristique $l=p$ positive [GMS, p.151].

Pour chaque nombre premier $l=2,3,5$, on considère respectivement le groupe $G$ déployé de type $G_{2}, F_{4}$ et $E_{8}$ et son $k$-sous-groupe

$$
A=\mu_{l} \times \mu_{l} \times \mathbb{Z} / l \mathbb{Z},
$$


avec les conventions de [GQM, §6.A]. On a

$$
H_{\text {fppf }}^{1}(k, A)=k^{\times} /\left(k^{\times}\right)^{l} \times k^{\times} /\left(k^{\times}\right)^{l} \times H^{1}(k, \mathbb{Z} / l \mathbb{Z}) ;
$$

tout élément est donc le produit de deux classes $(a),(b) \in k^{\times} /\left(k^{\times}\right)^{l}$ et d'un caractère $\chi \in H^{1}(k, \mathbb{Z} / l \mathbb{Z})$. On note $E_{a}$ le $\mu_{l}$-torseur $x^{l}=a$ pour $a \in k^{\times}$, et $E_{\chi}$ le $\mathbb{Z} / l \mathbb{Z}$-torseur associé au caractère $\chi$. Commençons par préciser le théorème 6.6 de $[\mathrm{GQM}]$ :

Théorème 2.1 (1) Le composé

$$
r_{G} \circ i_{k}: H_{f p p f}^{1}(k, A) \rightarrow H_{f p p f}^{1}(k, G) \rightarrow H^{3}(k),
$$

applique $(a) \times(b) \times \chi$ sur $-\chi \cup(a) \cup(b)$ si $l \in k^{\times}$et sur $\chi \cup(a) \cup(b)$ si $k$ est de caractéristique $l$.

(2) Si $E=E_{a} \times_{k} E_{b} \times_{k} E_{\chi}$, alors on a un isomorphisme

$$
Z_{\left({ }^{E} G\right)}\left(\mu_{l}^{(1)}\right) \stackrel{\sim}{\longrightarrow}\left(\mathrm{SL}_{1}(B) \times \mathrm{SL}_{1}(C)\right) / \mu_{l}
$$

où $B, C$ sont des $k$-algèbres cycliques de degré $l$ définies par

$$
\left.[B]=[C]=\chi \cup(b) \in \operatorname{Br}(k) \text { pour } G_{2} \text { (resp. } F_{4}\right) \text { et } l=2 \text { (resp. } 3 \text { ), }
$$
et $[B]=3[C]=\chi \cup(b) \in \operatorname{Br}(k)$ dans le cas $E_{8}$ et $l=5$.

L'assertion (1) est prouvée dans [GQM]; on raffine l'argument afin d'obtenir (2).

Démonstration : (2) On suppose que $G=E_{8}$ et $l=5$, les deux autres cas étant similaires. On a $Z_{G}\left(\mu_{5}^{(1)}\right)=H=\left(\mathrm{SL}_{5} \times \mathrm{SL}_{5}\right) / \mu_{5}$ avec les notations de [GQM, §6] et on souhaite décrire $Z_{\left(E_{G}\right)}\left(\mu_{5}^{(1)}\right)={ }^{E} H=\left(\mathrm{SL}_{1}(B) \times \mathrm{SL}_{1}(C)\right) / \mu_{5}$ pour des $k$ algèbres de degré 5 . Comme $H$ commute avec $\mu_{5}^{(1)}$, on a ${ }^{E} H={ }^{E^{\prime}} H$, où $E^{\prime}$ désigne le torseur $E^{\prime}=E_{b} \times E_{\chi}$. Pour déterminer ces algèbres, on peut par relèvement en caractéristique nulle supposer que $l$ est inversible dans $k$. De plus, on peut même supposer que $k$ contient une racine primitive $l$-ième de l'unité. Le résultat découle alors de la description de ${ }^{E^{\prime}} H$ donnée dans la preuve de l'assertion (1) [GQM]. 


\section{Groupes semi-simples anisotropes exotiques}

Proposition 3.1 On suppose que le corps de base est de caractéristique $p=2$ (resp. $p=3,5$ ) et que $H_{p}^{3}(k) \neq 0$. Alors il existe une $k$-forme $G^{\prime}$ anisotrope du groupe déployé $G$ de type $G_{2}$ (resp. $F_{4}, E_{8}$ ) qui contient un sous-groupe $R_{K / k}\left(\mathbb{G}_{m}\right) / \mathbb{G}_{m}$ où $K / k$ est une extension de corps (purement) inséparable de degré $p$.

Plaçons nous par exemple sur l'un des corps $\mathbb{F}_{p}\left(T_{1}, T_{2}\right)$ ou $\mathbb{F}_{p}\left(\left(T_{1}\right)\right)\left(\left(T_{2}\right)\right)$, qui satisfont l'hypothèse $H_{p}^{3}(k) \neq 0$. Comme $R_{K / k}\left(\mathbb{G}_{m}\right) / \mathbb{G}_{m}$ est un $k$-groupe unipotent lisse et connexe de dimension $p-1$ [CGP, 1.1.3], la proposition cidessus fournit des exemples de groupes anisotropes possédant des sous-groupes unipotents lisses et connexes non triviaux.

Lemma 3.2 Sous les hypothèses de la proposition 3.1, soit $E / k$ un $G$-torseur tel que $r_{p}([E]) \neq 0 \in H_{p}^{3}(k)$. Alors le $k$-groupe tordu ${ }^{E} G / k$ est anisotrope.

Démonstration. Comme la condition est préservée par extension finie de degré premier à $p$, il est loisible de supposer que $k$ ne possède pas d'extensions propres de degré premier à $p$. Il suffit alors de vérifier qu'une forme isotrope $G^{\prime}={ }^{E} G$ a un invariant de Rost trivial. Si $G^{\prime}$ est de type $G_{2}$, un tel groupe $G^{\prime}$ est déployé.

Dans le cas de type $F_{4}$, le noyau anisotrope de $G^{\prime}$ est trivial ou de type $B_{3}$, donc déployé puisque $\operatorname{cd}_{2}(k)=1$.

Si $G^{\prime}$ est de type $E_{8}$, le noyau anisotrope $J$ de $G^{\prime}$ est trivial ou intérieur de type $D_{4}, D_{6}, E_{6}, D_{7}, E_{7}$. Mais $\operatorname{cd}_{2}(k)=\operatorname{cd}_{3}(k)=1$, ce qui implique que $J$ est déployé (cf. [S, §4.4]). On conclut que $G^{\prime}$ est déployé.

Démonstration de la proposition 3.1. Le groupe $H_{p}^{3}(k) \neq 0$ est engendré par les classes décomposables, c'est-à-dire de la forme $(a) \cup(b) \cup \chi$. Il existe donc $a, b \in k^{\times}$et $\chi \in H^{1}(k, \mathbb{Z} / p \mathbb{Z})$ tel que $(a) \cup(b) \cup \chi \neq 0 \in H_{p}^{3}(k)$. Avec les notations du théorème 2.1.2, on note $E / k$ le $A$-torseur défini par $a, b$ et $\chi$. On considère le $k$-groupe $G^{\prime}={ }^{E} G$. Son invariant de Rost modulo $p$ est $(a) \cup(b) \cup \chi$ donc $G$ est anisotrope en vertu du lemme 3.2. En outre, par l'assertion (2) du théorème 2.1, 
$G^{\prime}$ contient le $k$-groupe

$$
H^{\prime}=\left(\mathrm{SL}_{1}(B) \times \mathrm{SL}_{1}(C)\right) / \mu_{p} .
$$

Si $p=2$ ou 3 , on a $B \cong C \cong(\chi, b)$ et $H^{\prime}$ contient $\operatorname{PGL}_{1}((\chi, b))$ par le plongement diagonal. Mais, comme on l'a déjà noté dans l'introduction, l'algèbre cyclique $(\chi, b)$ contient l'extension purement inséparable $K_{b}:=k(\sqrt[p]{b})$. D'où un plongement $R_{K_{b} / k}\left(\mathbb{G}_{m}\right) / \mathbb{G}_{m} \subset \mathrm{PGL}_{1}((\chi, b)) \subset H^{\prime} \subset G^{\prime}$.

Le cas $l=5$ est légèrement plus compliqué du fait que le sous-groupe maximal $H=\left(\mathrm{SL}_{5} \times \mathrm{SL}_{5}\right) / \mu_{5}$ ne contient pas $\mathrm{PGL}_{5}$ (en effet $\mu_{5}$ se plonge dans $\mu_{5} \times \mu_{5}$ suivant $\left.x \mapsto\left(x, x^{2}\right)\right)$. Cependant comme $B \cong(\chi, b)$ et $C \cong\left(\chi, b^{2}\right)$, on dispose de plongements naturels $j_{B}: R_{K_{b} / k}^{1}\left(\mathbb{G}_{m}\right) \rightarrow \mathrm{SL}_{1}(B)$ et $j_{C}: R_{K_{b} / k}^{1}\left(\mathbb{G}_{m}\right) \rightarrow \mathrm{SL}_{1}(C)$. On considère l'homomorphisme

$$
R_{K_{b} / k}^{1}\left(\mathbb{G}_{m}\right) \rightarrow\left(\mathrm{SL}_{1}(B) \times \mathrm{SL}_{1}(C)\right) / \mu_{5}, w \mapsto\left(j_{B}(w), j_{C}\left(w^{2}\right)\right)
$$

dont le noyau est $\mu_{5} \subset R_{K_{b} / k}^{1}\left(\mathbb{G}_{m}\right)$. On obtient ainsi un plongement

$$
R_{K_{b} / k}^{1}\left(\mathbb{G}_{m}\right) / \mu_{5} \cong R_{K_{b} / k}\left(\mathbb{G}_{m}\right) / \mathbb{G}_{m} \subset H^{\prime} \subset G^{\prime} .
$$

Remarque 3.3 Le premier $p$ est de "torsion" pour le groupe $G$ considéré. C'est une condition nécessaire pour obtenir de tels exemples. En effet, supposons que $p$ n'est pas de torsion pour un $k$-groupe $G$ semi-simple, simplement connexe et presque $k$-simple qui contient un sous $k$-groupe unipotent lisse $U$ non trivial. D'après Tits [T, 2.6], on sait alors que chaque élément de $U\left(k_{s}\right)$ est $k_{s}$-plongeable dans le radical unipotent d'un $k_{s}$-sous-groupe de Borel de $G$. Ceci entraîne que $U\left(k_{s}\right)$ est $k_{s}$-plongeable dans le radical unipotent d'un $k_{s}$-sous-groupe parabolique de $G$ [BT, 3.6]. Ainsi on peut associer à $U$ le $k$-sous-groupe parabolique $P(U)$ de $G$ (ibid) dont le radical unipotent contient $U$ ce qui montre que le groupe est isotrope.

\section{Références}

[BT] A. Borel, J. Tits, Eléments unipotents et sous-groupes paraboliques de groupes réductifs. I Invent. Math. 12 (1971), 95-104. 
[CGP] B. Conrad, O. Gabber, G. Prasad, Pseudo-reductive groups, Cambridge University Press (2010).

[EKLV] H. Esnault, B. Kahn, M. Levine, E. Viehweg, The Arason invariant and mod 2 algebraic cycles, J. Amer. Math. Soc. 11 (1998), 73-118.

[Ga] S. Garibaldi, Cohomological invariants, exceptional groups and Spin groups, Memoirs of the American Mathematical Society 937 (2009).

[GMS] S. Garibaldi, A. Merkurjev et J.-P. Serre, Cohomological invariants in Galois cohomology, University Lecture Series 28 (2003), American Mathematical Society.

[Gi] P. Gille, Invariants cohomologiques de Rost en caractéristique positive, K-Theory 21 (2000), 57-100.

[GQM] P. Gille et A. Quéguiner-Mathieu, Formules pour l'invariant de Rost, Algebra and Number Theory 5 (2011), 1-35.

[GiS] P. Gille et T. Szamuely, Central simple algebras and Galois cohomology, Cambridge Studies in Advanced Mathematics 101 (2006), Cambridge University Press.

[K] K. Kato, Galois cohomology of complete discrete valuation fields, Algebraic K-theory, Part II (Oberwolfach, 1980), pp. 215-238, Lecture Notes in Math. 967 (1982), Springer.

[S] J.-P. Serre, Sous-groupe finis des groupes de Lie, Séminaire Bourbaki 864 (1998-1999), Astérisque 332, Société Mathématique de France.

[T] J. Tits, Unipotent elements and parabolic subgroups of reductive groups. II, Algebraic Groups Utrecht (1986), Lecture Notes in Mathematics 1271 (1987), 265-284.

Philippe Gille

UMR 8552 du CNRS, DMA, Ecole Normale Supérieure, F-75005 Paris

E-mail : Philippe.Gille@ens.fr

Anne Quéguiner-Mathieu

Université Paris 13, Sorbonne Paris Cité, LAGA, CNRS (UMR 7539), F-93430

Villetaneuse, France

E-mail : queguin@math.univ-paris13.fr 